\title{
Different methods for handling the corner reflections of absorbing boundary condition in the numerical simulation of seismic waves \\ Xing Li
}

Department of Applied Mathematics, Shanghai Second Polytechnic University, Shanghai 201209, P.R.China

email: xingli@sspu.edu.cn

Keywords: seismic waves, CE, MTF, PML. Absorbing boundary conditions, Angular point

\begin{abstract}
In numerical simulation of wave equation, in a limited area to establish artificial boundary absorbing boundary conditions, the angular point on the calculation area of the processing is also cannot ignore a problem. According to CE, MTF, PML three different boundary conditions, using different angular point processing method, and through the numerical example comparing with different methods, better numerical results in practical application.
\end{abstract}

\section{Introduction}

In numerical simulation of seismic wave propagation, establish absorbing boundary conditions in limited area is a very important problem. In 1977, Clayton and Engquist [1] aim at sound wave equation of two-dimensional space put forward a series of different order of absorbing boundary conditions (CE). In 1994 Berenger put forward a new kind of absorbing boundary conditions namely Perfectly Matched Layer (PML)[2],the boundary conditions of almost zero reflection. In the early 1980's, Chinese scholars put forward the transmission theory and multiple transmission formula of [3], called the MTF. The point is, through the direct simulation of various one-way wave kinematics characteristics together to establish ABC. On this basis, the handling of angular point computing area is also a can't ignore the problem In view of the sound wave equation of two-dimensional half space, Engquist and Madjda using different order number CE boundary conditions, set up corresponding to two order absorbing boundary condition corner conditions.Based on MTF of a discrete form of angular point condition, the first-order discrete form $\mathrm{CE}$ angle point in the condition of equivalence. In order to further improve the accuracy of the condition of the discrete form of angular point to higher order. Application form of second-order displacement PML formula of calculating area of different segmentation to deal with angular point problem. According to different angular point processing method, the results of numerical experiment is given, the numerical results confirm the validity of the promotion.

\section{Introduction to the absorbing boundary conditions}

Consider rectangular coordinate system $(x, y)$ in $2 \mathrm{~d}$ standard acoustic wave equation: $\frac{\partial^{2} u}{\partial t^{2}}=c^{2}\left(\frac{\partial^{2} u}{\partial x^{2}}+\frac{\partial^{2} u}{\partial y^{2}}\right)$, (1.1) Clayton and Engquist put forward a series of absorbing boundary conditions with different accuracy:

The first-order approximate: $\frac{\partial u}{\partial x}+\frac{1}{c} \frac{\partial u}{\partial t}=0$,

The second order approximation: $\frac{\partial^{2} u}{\partial x \partial t}+\frac{1}{c} \frac{\partial^{2} u}{\partial t^{2}}-\frac{c}{2} \frac{\partial^{2} u}{\partial y^{2}}=0$

This is the famous Clayton-Engquist paraxial approximation (paraxial approximation) absorbing boundary conditions, the abbreviation CE.In the MTF boundary conditions, $\mathrm{N}$ order transmission formula[3]: 


$$
u_{0}^{p+1}=\sum_{j=1}^{N}(-1)^{j+1} C_{j}^{N} u_{j}^{p+1-j}
$$

Derivation for the formula (1.1), to get a new differential equation:

$$
\begin{aligned}
& \left(\partial_{t}+d(x)\right)^{2} u_{1}=c^{2} \frac{\partial^{2} u}{\partial x^{2}} \\
& \left(\partial_{t}+d(x)\right)^{3} u_{2}=-c^{2} d^{\prime}(x) \frac{\partial u}{\partial x} \\
& \partial_{t}^{2} u_{3}=c^{2} \frac{\partial^{2} u}{\partial y^{2}}
\end{aligned}
$$

In formula (1.3),make $d(x)=\frac{3 c}{2 \delta} \log \left(\frac{1}{R}\right)\left(\frac{x}{\delta}\right)^{2}$ here, $\delta$ is PML region width, $R$ is the reflection coefficient.

\section{CE and MTF corner conditions}

For two-dimensional half space of the acoustic wave equation (1.1) Engquist and Madjda CE boundary conditions using different order number, and put forward the corresponding to two order absorbing boundary condition corner conditions:

$\sqrt{2} \frac{\partial u}{\partial t}+\frac{\partial u}{\partial x}+\frac{\partial u}{\partial y}=0 \quad,(2.1),(x, y)$ is the angular point area, $t \geq 0$.

Alain Bamaberger given another corner conditions in 1990 [4]: $\gamma \frac{\partial u}{\partial t}+\frac{\partial u}{\partial x}+\frac{\partial u}{\partial y}=0,(x, y)$ is the angular point area, $t \geq 0$. where, $\gamma$ is a constant. Respectively using the first order and second order $\mathrm{CE}$ and the angular point above conditions near the corner regions of two-dimensional acoustic equation to carry on the numerical simulation, confirmed $\gamma$ take different values, the calculation accuracy have great influence. For example, $\gamma$ separately equal to $1.5,0.1$ and $3.0, \gamma=1.5$ is obtained by numerical experiments, the best close to the exact solutions of the (consistent with his theory), And points out that when $\gamma=\sqrt{2}$, the numerical results and almost no difference between $\gamma=1.5$. So imagine a discrete form based on MTF of corner conditions, and points out that the first order discrete form of angular point condition and CE angular point of equivalence. In order to further improve the accuracy of the condition of the discrete form of angular point to higher order.

Already in the artificial boundary $\Gamma_{1}$ and $\Gamma_{2}$ established $N$ order MTF, as shown in figure (2.1). In a corner on the 45 degree Angle in the direction of the straight line of first-order MTF is established,In this case, the formula and the formula (1.2) the same, but the formula (1.2) step to the space $\sqrt{2} \Delta x$. It can be shown in a straight line at 45 degrees built this discrete corners and corner conditions of the conditional expression (2.1) is equivalent to. Certify as follows: the $x, y$ axis rotated 45 degrees counter-clockwise direction to give the new axes, in Figure 1 below. In this case the coordinate transformation:

$$
\begin{aligned}
& x=x^{\prime} \cos \theta-y^{\prime} \sin \theta, \\
& y=y^{\prime} \cos \theta+x^{\prime} \sin \theta,
\end{aligned}
$$

$$
\text { here, } \theta=45^{\circ} \text {. }
$$

Under the new coordinate system, a step at both ends of the formula (1.2) of the MTF conditions $u_{0}^{p+1}=u_{1}^{p}(2.3)$ make a Taylor expansion at the point at $\left(t, x^{\prime}\right)=(p \Delta t, 0)$ and get: 


$$
\begin{aligned}
& u(p \Delta t+\Delta t, 0)=u(p \Delta t, 0)+\frac{\Delta t}{1 !} \frac{\partial u}{\partial t}+o(\Delta t), \\
& u\left(p \Delta t,-c_{a} \Delta t e_{1}\right)=u(p \Delta t, 0)+\frac{\left(-c_{a} \Delta t\right)}{1 !} \frac{\partial u}{\partial x^{\prime}}+o\left(c_{a} \Delta t\right),
\end{aligned}
$$

Thus, the Formula $u_{0}^{p+1}=u_{1}^{p}$ change to $\frac{\partial u}{\partial t}+\frac{\partial u}{\partial x^{\prime}}=0$, Re-use formula (2.2) in two type of formula (2.1). In order to further improve the accuracy of the first-order discrete corner aforementioned conditions be extended to higher order. Thus, in a straight line through the corners at 45 degrees higher order MTF. That is using the formula (1.2), then for the corresponding interpolated time or space.

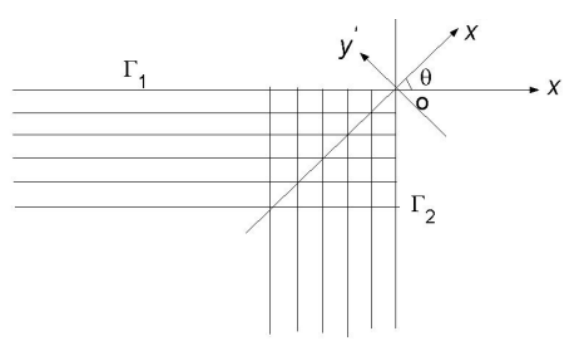

Fig2.1 MTF calculating model

\section{Processing PML boundary corner points}

When calculated on the border area with PML absorbing boundary conditions, the area is divided into regions and PML calculation area. As shown in Figure 3.1 left.Where, $\Omega$ is calculate region, $\Omega_{1}, \Omega_{2}, \Omega_{3}, \Omega_{13}, \Omega_{23}$ is the PML region. In Equation 1.3, the right-hand side of the solution $u$ is defined on the entire region $\Omega+\Omega_{1}+\Omega_{2}+\Omega_{3}+\Omega_{13}+\Omega_{23}$, and left in subparagraph $u_{1}, u_{2}, u_{3}$ defined in the PML region $\Omega_{1}+\Omega_{2}+\Omega_{3}+\Omega_{13}+\Omega_{23}$. Therefore, in equation (1.3) discrete, discrete left border over the area $\Omega_{1}+\Omega_{13}, \Omega_{2}+\Omega_{23}$ right boundary in the area of discrete, discrete on the bottom border region $\Omega_{13}+\Omega_{3}+\Omega_{23}$, which is a method for segmentation. Another division method as shown in Figure 3.1 right, when equation (1.3) discrete, discrete left border on $\Omega_{1}, \Omega_{2}$ on the right border of discrete, discrete bottom border on $\Omega_{3}$. On two different discrete way, we will confirm the first discretely reflection will occur at the corner points of the numerical examples below, while the second will be at the corner discretely handled well, almost no reflection.
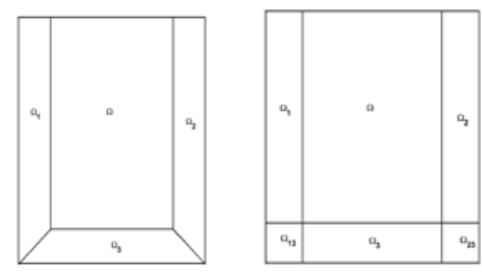

Figure 3.1 calculation region and PML region

(1) In the numerical example, since the second order in 1 CE-order boundary conditions:

$$
\frac{\partial u}{\partial n}+\frac{1}{c} \frac{\partial u}{\partial t}=0
$$

Processing for boundary $\Gamma$. Where $n$ is the normal vector boundary. Discrete finite difference, such as the right boundary for differential discrete form, formula (3.1) is

$u_{i+1, j}^{n+1}=u_{i+1, j}^{n}-c \frac{\Delta t}{\Delta x}\left(u_{i+1, j}^{n}-u_{i, j}^{n}\right)$.

(2) For multi-transmitting formula MTF, we use spatial interpolation ways. 
(3) In the finite difference method for PML formula discrete. We use five-point difference format commonly used.

\section{Numeral Calculations}

To further validate the corner conditions mentioned above, we consider a two-dimensional focus on issues close to the corner reflection.Take the calculation region $\Omega=[0,1] \times[0,1], \Gamma_{1}, \Gamma_{2}$ is an artificial boundary, shown in Figure 4.1. In order to cause strong reflection near the corner point, the focal point on the upper right corner of the area $(0.89,0.89)$ at the source radius is 0.04 distributed force source. When meshing take space step is $\Delta x=\Delta y=0.0025$, a time step of $\Delta t=0.00125$. The left end of the equation (1.1) of the central difference, using the five-point difference right format commonly used, the overall use of explicit discrete format. The exact solution by far is set artificial boundaries. 1-4 respectively in order MTF condition numerical calculation. Firstly, given the time $t$ of snapshots. Figure 4.2 is a snapshot . Figure 4.2a is where $t=0.5 \mathrm{~s}$ time of exact solutions of the wave field snapshots Figure 4.2b- Figure 4.2e is MTF and the corresponding corner of the aforementioned conditions of use calculated numerical solution of $t=0.5 \mathrm{~s}$ when the wave field snapshot . With the improvement of MTF order of increasing numerical solution with the exact solution to better fit 。Can be seen from the figure, the third-order condition is obviously superior to the first-order and second-order conditions, numerical and exact solutions of Fourth Order MTF and corresponding corner conditions coincide.

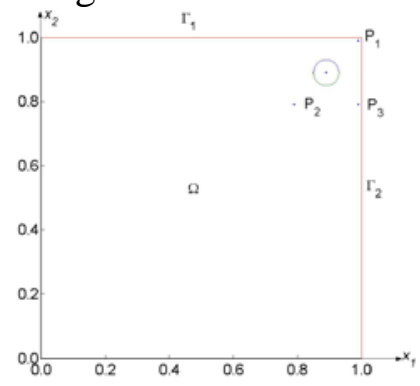

Fig 4.1 calculation area and source location

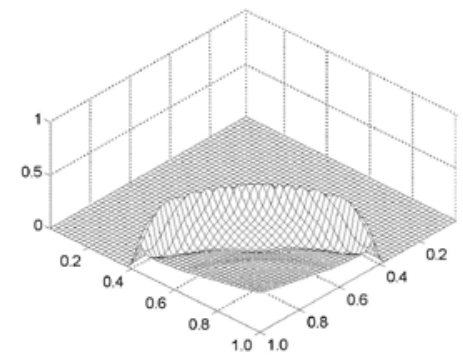

Fig 4.2b first-order conditions at $t=0.5 \mathrm{~s}$

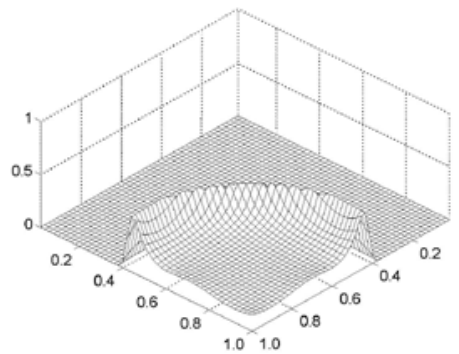

Fig4.2d third-order conditions at $t=0.5 \mathrm{~s}$

Here is the numerical results for the above process is the opposite corner of the area with the PML absorbing boundary conditions. Area size is $1000 \times 1500 m, \Delta x=\Delta y=5 m$, $\Delta t=0.0005 \mathrm{~s}, c=2000 \mathrm{~m} / \mathrm{s}$, In the bottom right corner of Gauss source added. Internal area with

Fig4.2a precise solution at $t=0.5 \mathrm{~s}$

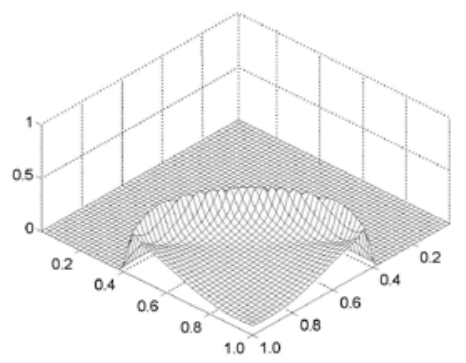

Fig4.2c second-order conditions at $t=0.5 \mathrm{~s}$

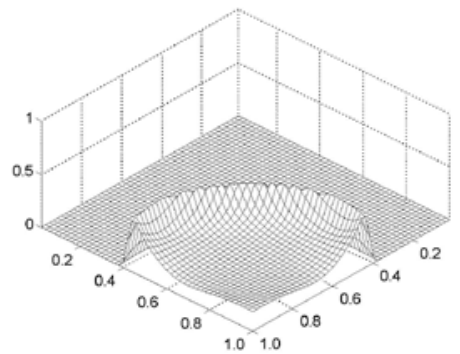

Fig 4.2e fourth-order conditions at $t=0.5 \mathrm{~s}$ 
double quadratic quadrilateral element calculation, PML region with discrete difference.
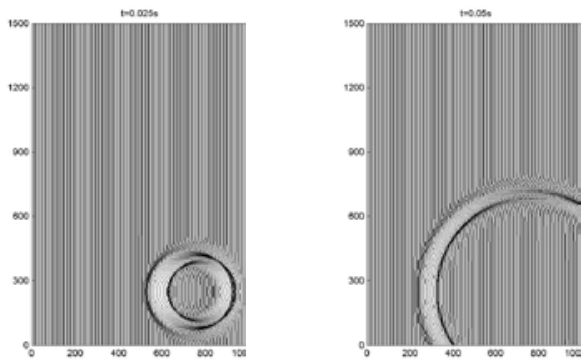

Fig4.3 wave field snapshots
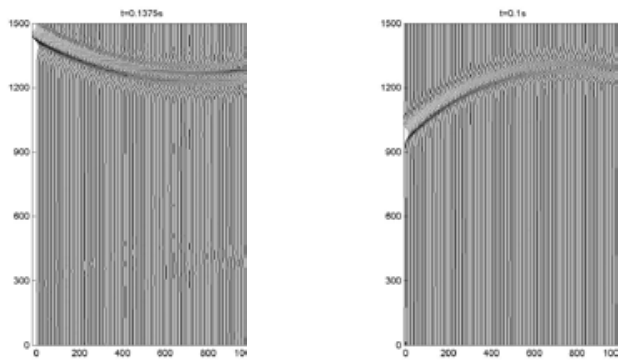

Fig 4.4. wave field snapshots

In the above diagram, Figure 4.3 is time-wave has not yet reached the border; Figure 4.4 is the wave reaches the boundary, the first is the processing method in Figure 3.1 PML region. Figure 3.2 is the second approach to the PML region. As can be seen from the figure, the process by the first method, the corner points will be reflected; while the second method for processing, corner points almost no reflection.

\section{References}

[1] W. D. Smith ,A non-reflecting plane boundary for wave propagation problems ,J.Comput. Phys.1974,15:492-503.

[2] Berenger, J. P., Three-dimensional perfectly matched layer for the absorption of electromagnetic waves , J. Comput. Phys., 1994,127: 363-379.

[3] Chew, W. C. \&Liu, Q., Perfectly matched layers for the electrodynamics: a new absorbing boundary condition, J. Comp. Acoust., 1996, 4:341-359.

[4] Siam J. Number. Anal, Second-order absorbing boundary conditions for the wave equation: A solution for the corner problem,1990, 27: 323-352.

[5] Liao Z P and H L Wong, A transmitting Boundary for the Numerical Simulation of Elastic Wave Propagation, Soil Dyn. Earthq. Eng.,1984,3:174-1. 